\title{
Ground-glass opacity: High-resolution computed tomography and 64-multi-slice computed tomography findings comparison
}

\author{
Gianluigi Sergiacomi*, Carmelo Cicciò, Luca Boi, Luca Velari, Sonia Crusco, \\ Antonio Orlacchio, Giovanni Simonetti
}

Department of Diagnostic Imaging, Molecular Imaging, Interventional Radiology and Radiation Therapy,

University Polyclinic of Tor Vergata, Viale Oxford 81, 00133 Rome, Italy

\section{A R T I C L E I N F O}

\section{Article history:}

Received 24 November 2008

Accepted 18 March 2009

\section{Keywords:}

Ground-glass opacity

High-resolution computed tomography

Volumetric computed tomography by

64-row multi-slice scanner

\begin{abstract}
A B S T R A C T
Objective: Comparative evaluation of ground-glass opacity using conventional high-resolution computed tomography technique and volumetric computed tomography by 64-row multi-slice scanner, verifying advantage of volumetric acquisition and post-processing technique allowed by 64-row CT scanner.

Methods: Thirty-four patients, in which was assessed ground-glass opacity pattern by previous highresolution computed tomography during a clinical-radiological follow-up for their lung disease, were studied by means of 64-row multi-slice computed tomography. Comparative evaluation of image quality was done by both CT modalities.

Results: It was reported good inter-observer agreement ( $k$ value $0.78-0.90)$ in detection of ground-glass opacity with high-resolution computed tomography technique and volumetric Computed Tomography acquisition with moderate increasing of intra-observer agreement ( $k$ value 0.46 ) using volumetric computed tomography than high-resolution computed tomography.

Conclusions: In our experience, volumetric computed tomography with 64-row scanner shows good accuracy in detection of ground-glass opacity, providing a better spatial and temporal resolution and advanced post-processing technique than high-resolution computed tomography.
\end{abstract}

(c) 2009 Elsevier Ireland Ltd. All rights reserved.

\section{Introduction}

The interstitial-alveolar oedema, featuring as a ground-glass opacity (GGO) in lung computed tomography (CT), is characterized by an area of slightly increased attenuation, caused by alveolar collapse, interstitial thickening and partial filling of air spaces, due to the presence of fluids, inflammatory cells and cellular debris with preservation of bronchial and vascular structures [1].

First described by Klein and Gamsu [2], GGO is considered a non-specific finding and may be representative of a wide spectrum of diseases from inflammatory and infective to neoplastic. However, it is usually associated with other features such as nodules, areas of consolidation and interstitial abnormalities that may contribute to the specificity of diagnosis. Well-known examples are the 'halo sign' in invasive aspergillosis and the 'crazy-paving' in alveolar proteinosis [3,4].

Furthermore, lobular distribution of the GGO can help obtain a preliminary diagnosis: e.g. centrolobular in hypersensitivity pneumonitis or panlobular in Pneumocystis carinii pneumonia [5,6].

\footnotetext{
* Corresponding author. Tel.: +39 06 20902401; fax: +39 0620902404.

E-mail address: carmeciccio@libero.it (G. Sergiacomi).
}

Many studies have shown that high-resolution CT (HRCT) is the technique of choice in the study of the chronic diffuse infiltrative lung disease $[7,8]$. This is due to its accuracy in showing GGO during both the early stages of disease and follow-up, thus offering the opportunity to start adequate therapies to prevent a possible fibrotic evolution.

The study of the secondary pulmonary lobule, in which we observe many features of diffuse lung disease, is usually performed with HRCT that allows high spatial resolution $(0.5 \mathrm{~mm})$ with ideal assessment of high intrinsic contrast structures such as the lung parenchyma.

Although HRCT with discontinuous thin sections acquisitions is the technique of choice for the study of diffuse lung diseases, multi-slice CT (MSCT) with volumetric acquisitions has become the technique of choice in detecting focal pulmonary abnormalities. MSCT allows an optimization of the diagnostic content of CT with shorter acquisition times, thinner sections and the possibility of 2D and $3 \mathrm{D}$ reconstructions not obtainable with HRCT discontinuous acquisition.

The advent of the 64-row MSCT has substantially improved the advantages of this system resulting both in a faster scanning (4-6s) for the chest and a higher spatial resolution $(0.35 \mathrm{~mm})$ thus achieving an isotropic voxel sampling on the $z$-axis [9]. 

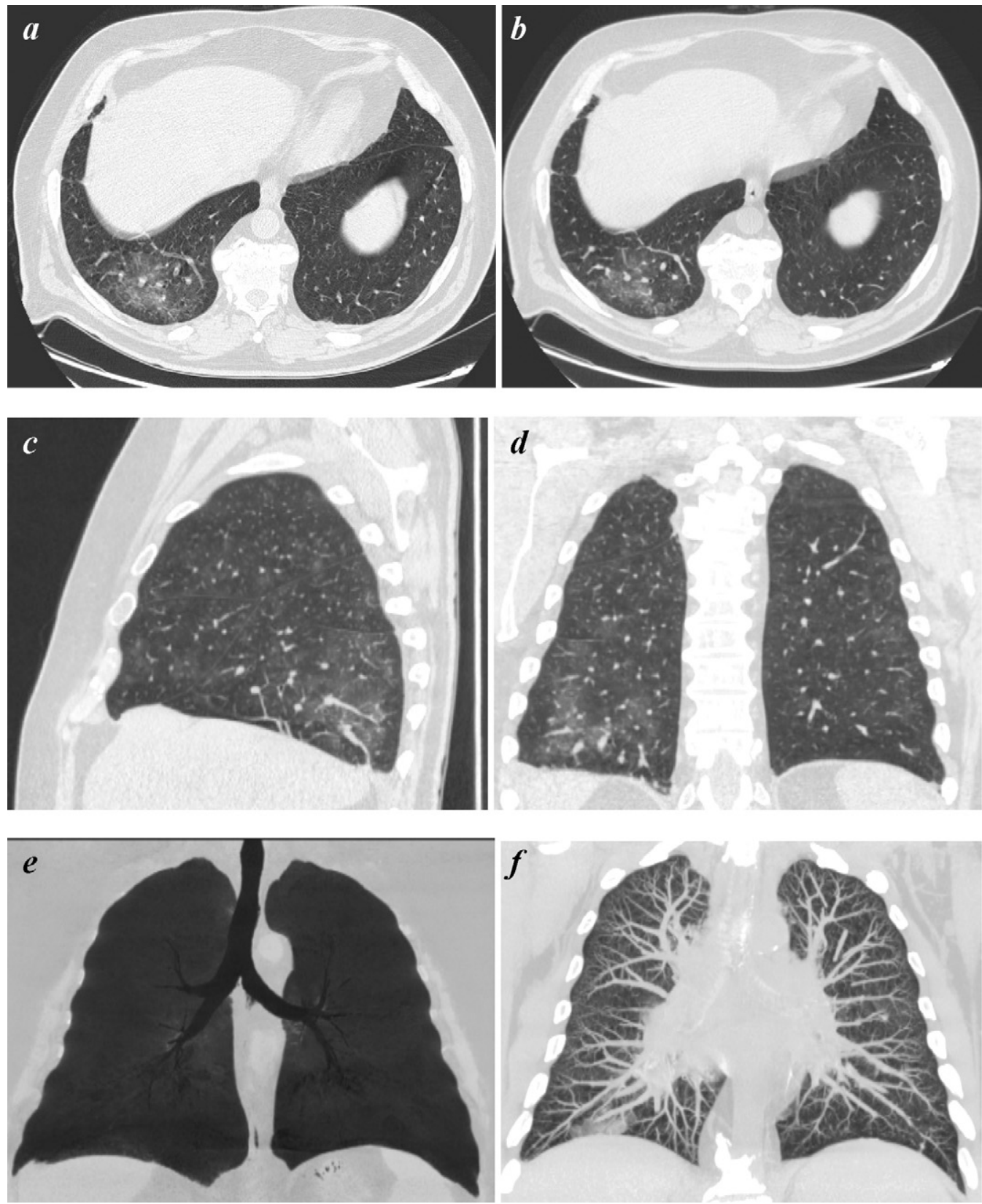

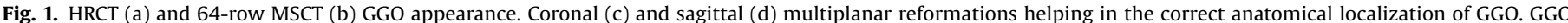
displayed with MIP (e) and mIP (f).

The faster scanning ability allows the study of uncooperative patients therefore reducing cardio-respiratory artefacts.

Purpose of our study was to compare HRCT and MSCT in the assessment of GGO in diffuse lung diseases.

\section{Methods}

\subsection{Population}

From January 2006 to December 2007, thirty-four patients (21 males, 13 females; range 20-72 years; mean-age 46 years) with a previous HRCT showing a ground-glass pattern and needing a strict follow-up, were consecutive studied by 64-row MSCT (LightSpeed VCT 64 slice, General Electric, Milwaukee, WI, USA) and with a CT protocol routinely used in our Department.
The patients were affected by idiopathic pulmonary fibrosis (IPF) (seven males, three females), Hodgkin's Lymphoma (five males, five females), sarcoidosis (five males, three females), eosinophilic pneumopathy (four males, two females).

An informed consent was obtained from all patients.

\subsection{CT protocol}

\subsection{1. $H R C T$}

HRCT studies (Figs. 1a and 2a) consisted of 20-25 s scanning of a series of non-contiguous $1.25 \mathrm{~mm}$ thick slices (step-by-step acquisition), at $10 \mathrm{~mm}$ intervals, using a high-spatial-frequency reconstruction algorithm and standard settings $(120 \mathrm{kV}, 220 \mathrm{~mA}$ and rotation time of $0.5 \mathrm{~s}$ ). The patient was in supine position head first and four slices per tube rotation from the apices to the lung bases were obtained during a single breath-hold. 

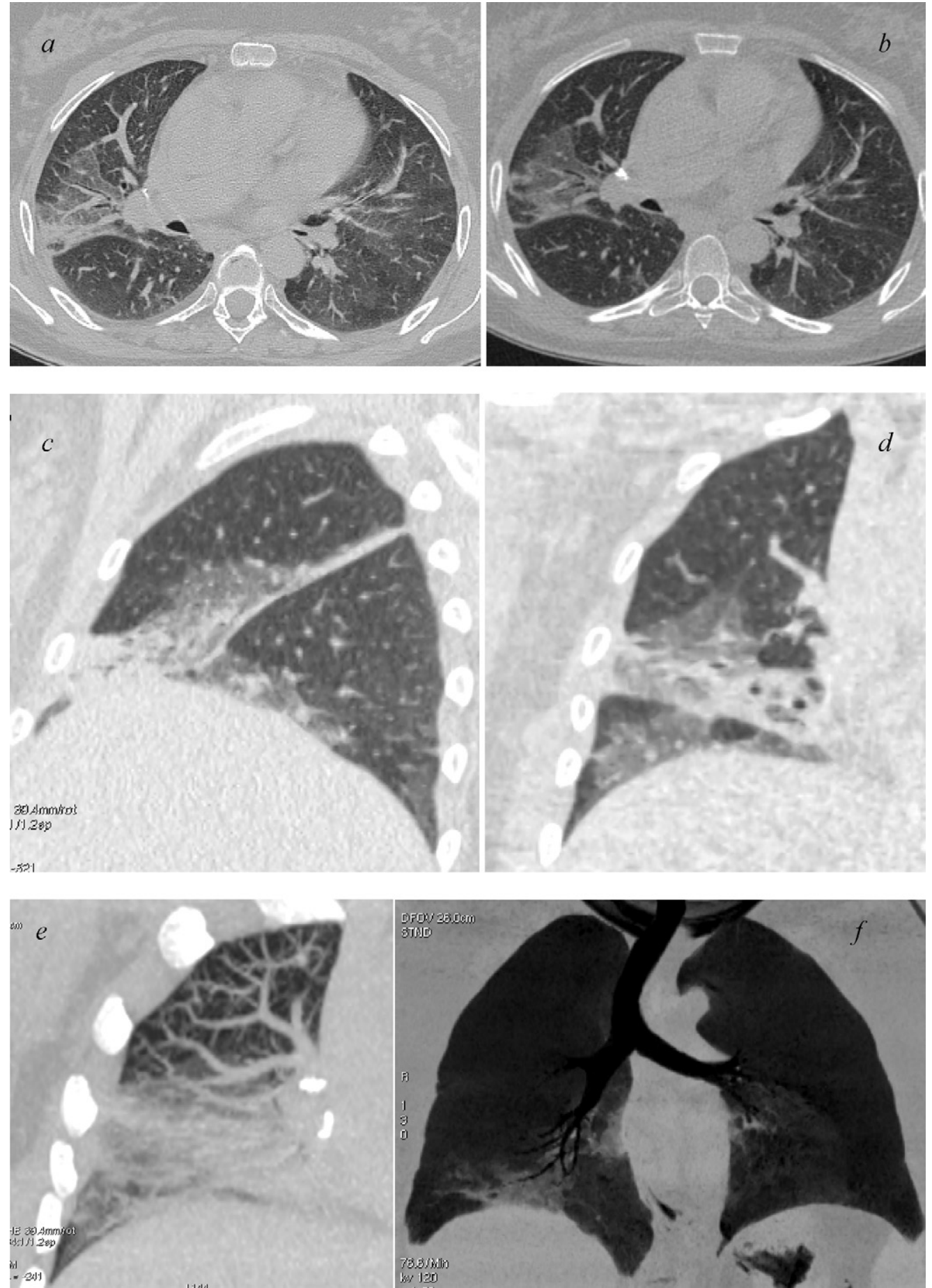

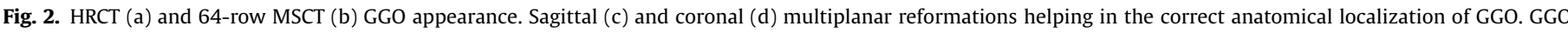
displayed with MIP (e) and MIP (f).

The effective radiation doses were $0.98 \mathrm{mSv}$.

\subsection{2. $M S C T$}

MSCT acquisitions were acquired in mean time of 2 months after HRCT study.

MSCT acquisitions (Figs. $1 \mathrm{~b}$ and $2 \mathrm{~b}$ ) consisted in contiguous whole lung scanning from the lung apices to the bases using a 64row MSCT scanner, with $5 \mathrm{~mm}$ collimation, $0.4 \mathrm{~s}$ rotation time and table speed of $55 \mathrm{~mm} / \mathrm{s}$.

The patient was in supine position head first and the images were acquired during a single breath-hold in $4 \mathrm{~s}$, using a low radi- ation dose thanks to the natural contrast of the lung and using the Smart-mA protocol for automatic modulation of exposure $(120 \mathrm{kV}$, 100-350 smart $\mathrm{mA}$ ) [10].

The effective radiation doses were $2.9 \mathrm{mSv}$.

\subsection{Image analysis}

After the volumetric scanning, image data set were reconstructed with slice thickness/reconstruction interval: $1.25 / 0.625 \mathrm{~mm}$ and high-resolution kernel. 
Table 1

Intra-observer agreement in the assessment of GGO using an HRCT and a 64-row MSCT scanner.

\begin{tabular}{ll}
\hline Intra-observer agreement $k$ value & HRCT/MSCT \\
\hline Radiologist A & 0.63 \\
Radiologist B & 0.75 \\
Radiologist C & 0.83
\end{tabular}

a $k$ Values: $\leq 0.20$, poor; $0.21-0.40$, fair; $0.41-0.60$, moderate; $0.61-0.80$, good; $\geq 0.81$, very good.

Table 2

Inter-observer agreement in the assessment of GGO using an HRCT and a 64-row MSCT scanner.

\begin{tabular}{lll}
\hline Inter-observer agreement $k$ value & HRCT & MSCT \\
\hline Radiologist A vs. B & 0.39 & 0.54 \\
Radiologist B vs. C & 0.46 & 0.46 \\
Radiologist A vs. C & 0.78 & 0.90
\end{tabular}

a $k$ Values: $\leq 0.20$, poor; $0.21-0.40$, fair; $0.41-0.60$, moderate; 0.61-0.80, good; $\geq 0.81$, very good.

Abnormal findings were better assessed by obtaining multiplanar reformations (2D, $1.25 \mathrm{~mm}$ thick) on the sagittal (Figs. 1c and 2c) and coronal plane (Figs. 1d and 2d).

In addition, MIP (maximum intensity projection) and mIP (minimum intensity projection) post-processing techniques were applied. This allowed creating a bi-dimensional image of voxels with higher (MIP) (Figs. 1f and 2f) or lower (mIP) density (Figs. 1e and $2 \mathrm{e}$ ) thus enhancing the differences between the lung structures.

The large number of images produced was independently evaluated on a workstation (Advantage 4.2, General Electric) with lung window setting (width $1500 \mathrm{HU}$; level - 500/650 HU) by three radiologists with at least 10 years of experience in chest radiology, aware of the clinical condition of the patients, but blinded to the technique of image acquisition.

The radiologists were subsequently asked to evaluate the sharpness of the abnormal lung features particularly the GGO, but also the associated reticular patterns, the small nodules, the areas of decreased lung attenuation and the bronchiectasis. The images of each patient were evaluated in random order and retrospectively with the interval of 1 month.

A qualitative judgment of GGO representations in both HRCT and MSCT techniques was scored by a scale from 0 (worst) to 4 (best).

\subsection{Statistical analysis}

The statistical analysis was performed by comparing the qualitative judgment expressed by the three reviewers and the diagnostic results provided by both $\mathrm{CT}$ techniques, to establish the agreement rate among the observers. Agreement between (inter) and within (intra) observers was assessed using the Cohen's $k$ statistic [11] (Tables 1 and 2).

The value of $k$ was: $0-0.20$ poor agreement; $0.21-0.40$ fair agreement; $0.41-0.60$, moderate agreement; $0.61-0.80$ good agreement; 0.81-1.00 excellent agreement.

\section{Results}

Statistical analysis showed an overlapping of the diagnostic judgment independently expressed by the three radiologists by using HRCT images and MSCT images (Table 1) (Figs. 1 and 2a,b).

A better inter-observer agreement in the diagnostic judgement was noticed when using MSCT rather than HRCT images in detection of GGO: radiologist $\mathrm{A}$ and $\mathrm{B}$ (from fair to moderate: $k$ value $0.29-0.54$ ); radiologist $\mathrm{A}$ and $\mathrm{C}$ (from good to excellent: $k$ value $0.78-0.90)$.
Only a moderate inter-observer agreement ( $k$ value 0.46$)$ was observed in the evaluation of images obtained with both techniques between radiologist $\mathrm{B}$ and $\mathrm{C}$ (Table 2 ).

The assessment of GGO has been obtained with high accuracy by both the techniques, without observing significant statistical difference $(P<0.002)$ between HRCT and MSCT in their detection.

Moreover, the multiplanar reconstructions of MSCT has allowed an easy identification of other features GGO associated such as nodules, areas of consolidation and interstitial abnormalities, contributing therefore to an increase of accuracy of diagnosis

\section{Discussion}

GGO is a common but non-specific feature of lung CT, representative of a wide spectrum of diseases in which it may be expression of an early damage either interstitial or alveolar [12].

GGO is mostly associated with diffuse pneumonias such as in opportunistic infections, with interstitial inflammatory diseases and with acute alveolar diseases. It can also be expression of a heterogeneous group of disorders such as drug-induced lung toxicity and neoplastic diseases.

In some cases, the appearance of GGO correlates with disease activity preceding irreversible changes such as fibrosis or 'honey combing' [12].

However, during the early stages an adequate treatment can reverse the cause of GGO. It is therefore of the utmost importance to provide a reliable and sensitive imaging technique to correctly detect and monitor GGO evolution based on its extension and topographic distribution [13].

HRCT is to date recommended as the 'gold-standard' technique for the study of the secondary lobule, and is the most sensitive for the detection of GGO.

Remy-Jardin et al. [14] established that HRCT is more sensitive than conventional CT in detecting GGO and both fine bronchial and parenchymal lesions. More recently Studler et al. have confirmed the superiority of HRCT in the study of GGO compared to 16-row MSCT [15].

However, with the recent advent of the 64-row MSCT (Figs. 1 and $2 \mathrm{~b}$ ), the volumetric acquisition has become competitive with HRCT in detecting diffuse lung diseases [15].

MSCT acquisitions allow an optimization of the diagnostic content due to shorter scanning times, reduced artefacts and increased scan range per rotation on the $z$-axis, obtaining $0.625 \mathrm{~mm}$ thick sections and an isotropic dataset that allows multiplanar and 3D reconstructions with diagnostic quality comparable to HRCT (Figs. 1 and 2a) [16].

In addition to axial images we have used multiplanar reconstructions in sagittal (Figs. 1 and 2c) and coronal planes (Figs. 1 and 2d). This allowed an accurate topographic localization of the distribution of the pulmonary abnormalities, often crucial for a correct diagnosis.

Moreover, volumetric acquisitions allowed the application of MIP or mIP algorithms that are increasingly used in the detection of diffused lung diseases [17-19] (Figs. 1 and 2e and f).

In particular, mIP algorithm (Figs. 1 and $2 \mathrm{f}$ ) improves the detection of GGO by enhancing the difference between the low density of the airways and the higher density of the GGO. It also better demonstrate the bronchiectasis detected at HRCT [18].

In our study the mean duration of the chest scan was $4 \mathrm{~s}$, thus slightly shorter than in Studler et al. ( $4 \mathrm{~s}$ vs. $6 \mathrm{~s}$ ). This reduced the motion artefacts in poorly collaborating patients without decrementing the diagnostic content of the images acquired.

Moreover, as recommended by Remy-Jardin et al. [17], and benefiting from an isotropic dataset, we have performed multiplanar reconstruction in sagittal and coronal planes with a diagnostic and iconographic quality comparable to the axial images. 
Multiplanar rendering in fact allows a better evaluation of those pulmonary abnormalities, such as the apical lesions, that are located in areas more difficult to evaluate on the axial images provided by HRCT [17].

Our MSCT protocol included a slice thickness of $5 \mathrm{~mm}$ (vs. $1.5 \mathrm{~mm}$ used by Studler et al.) and a $1.25 \mathrm{~mm}$ (vs. $3 \mathrm{~mm}$ ) thick reconstruction algorithm. The option of using a thinner $0.6 \mathrm{~mm}$ slice reconstruction algorithm was discarded. This would have generated a great amount of images, difficult to manage and with a less diagnostic quality due to decreased signal/noise ratio.

In contrast to Studler et al. [15], we did not observe a significant statistical difference between HRCT and MSCT in detection of GGO (Table 1). Furthermore, statistical analysis demonstrated an increased inter-observer agreement by using volumetric acquisitions with reconstruction algorithms (MPR, MIP and MIP), compared to the axial evaluation alone of HRCT (Table 2).

The lower accuracy described by Studler et al. in detecting GGO by MSCT compared to HRCT could be due to the use of $3 \mathrm{~mm}$ reconstructions. In our study, the use of $1.25 \mathrm{~mm}$ and of high quality reconstruction algorithms (MPR, MIP and mIP), provided by the volumetric 64-row MSCT, improved the quality of the images, crucial for a correct diagnosis.

The increased number of detectors and their smaller size, grants an improved sensitivity and specificity in the detection of the main pulmonary anomalies and especially of GGO [9].

Our results, only supposed by Studler et al., are obtained thanks to the 64-row MSCT that is able to provide diagnostic images comparable to those obtained by HRCT.

Our choice of a $5 \mathrm{~mm}$ slice thickness with a $1.25 \mathrm{~mm}$ reconstruction, offers a good compromise between low dose, short scanning times and images of good diagnostic quality with a thickness similar to that of HRCT.

The main difference between MSCT and HRCT technique is the difference in dose emitted.

Our measures are slightly lower than those described in the literature for volumetric acquisitions $(0.98 \mathrm{mSv}$ for $\mathrm{HR}$ techniques and $2.9 \mathrm{mSv}$ for volumetric techniques) and evaluated following the European Union guidelines "16262 EN" [20]. In cases of immunodepressed or diffused lung disease patients, undergoing routine CT monitoring, low dose volumetric acquisitions similar to those described in the literature for HR, could be used to combine both the advantage of volumetric data and reduce patient exposure [21]. With this purpose our Department is performing a comparative qualitative and dosimetric study between HRCT and MSCT both performed using a low dose (50 mAs).

\section{Conclusions}

Volumetric computed tomography with 64-row scanner performed by $5 \mathrm{~mm}$ slice thickness and a $1.25 \mathrm{~mm}$ high-resolution reconstruction, shows a good imaging quality in detection of ground-glass opacity, compared to high-resolution computed tomography.

Reconstructed high-resolution axial images generated from MSCT are of comparable quality to images obtained using conventional axial high-resolution CT, allowing further high quality and effective algorithms of post-processing (MPR, MIP mIP).

Our experience moreover proves a usefulness of MSCT on detection of other pathologic features, such as nodules, areas of con- solidation and interstitial abnormalities, usually associated with GGO.

\section{Conflict of interests}

We do not prior or duplicate publication or submission elsewhere of any part of the study; the manuscript has been read and approved by all the authors and the criteria for authorship have been met; there are no financial or other conflict of interests; there are no suggestions such as referring possible unqualified reviewers due to conflict of interests. We submit our article using the online submission system.

\section{References}

[1] Austin JHM, Muller NL, Friedman PJ, et al. Glossary of terms for CT of the lungs: recommendations of the Nomenclature Committee of the Fleischner Society. Radiology 1996;200:327-31.

[2] Klein JS, Gamsu G. High-resolution computed tomography of diffuse lung disease. Invest Radiol 1989;24:805-12.

[3] Kuhlman J, Fishman E, Siegelman SS. Invasive pulmonary aspergillosis in acute leukemia: characteristic findings on $\mathrm{CT}$, the $\mathrm{CT}$ halo sign, and the role of $\mathrm{CT}$ in early diagnosis. Radiology 1985;157:611-4.

[4] Godwin JD, Muller NL, Takasugi JE. Pulmonary alveolar proteinosis: CT findings. Radiology 1988;169:609-13.

[5] Gruden JF, Webb WR. Identification and evaluation of centrilobular opacities on high-resolution CT. Semin Ultrasound CT MR 1995;16:435-49.

[6] Hansell DM, Wells AU, Padley SPG, et al. Hypersensitivity pneumonitis: correlation of individual CT patterns with functional abnormalities. Radiology 1996;199:123-8.

[7] Zerhouni EA, Naidich DP, Stitik FP, et al. Computed tomography of the pulmonary parenchyma. Part 2. Interstitial disease. J Thorac Imaging 1985;1:54-64.

[8] Mathieson JR, Mayo JR, Staples CA, et al. Chronic diffuse infiltrative lung disease: comparison of diagnostic accuracy of CT and chest radiography. Radiology 1989;171:111-6.

[9] Kohl G. The evolution and state-of-the-art principles of multislice computed tomography. Proc Am Thorac Soc 2005;2:470-6.

[10] Kalra MK, Rizzo S, et al. Chest CT performed with Z-axis modulation: scanning protocol and radiation dose. Radiology 2005;237:303-8.

[11] Cohen J. A coefficient of agreement for nominal scales. Educ Psychol Meas 1960;20:37-46.

[12] Muller NL, Staples CA, Miller AR, et al. Disease activity in idiopathic pulmonary fibrosis: CT and pathologic correlation. Radiology 1987;165:731-4.

[13] Vedal S, Welsh EV, Miller AR, et al. Desquamative interstitial pneumonia: computed tomographic findings before and after treatment with corticosteroids. Chest 1988;93:215-7.

[14] Remy-Jardin M, Remy J, Deffontaines C, et al. Assessment of diffuse infiltrative lung disease: comparison of conventional CT and high-resolution CT. Radiology 1991;181:157-62.

[15] Studler U, Gluecker T, Bongartz G, et al. Image quality from high-resolution $\mathrm{CT}$ of the lung: comparison of axial scans and of sections reconstructed from volumetric data acquired using MDCT. AJR 2005;185:602-7.

[16] Beigelman-Aubry C, Hill C, Grenier PA. Multi-detector row CT and postpro' cessing techniques in the assessment of diffuse lung disease. RadioGraphics 2005;25:1639-52.

[17] Remy-Jardin MJ, Campistron P, Amara A, et al. Workflow issue with multislice CT of the thorax: usefulness of multiplanar reformations in the diagnostic approach of infiltrative lung disease. Eur Radiol 2002;12(Suppl. 1): 134.

[18] Bhalla M, Naidich DP, McGuinness G, et al. Diffuse lung disease: assessment with helical CT-preliminary observations of the role of maximum and minimum intensity projection images. Radiology 1996;200:341-7.

[19] Remy-Jardin M, Remy J, Gosselin B, et al. Sliding thin slab, minimum intensity projection technique in the diagnosis of emphysema: histopathologic-CT correlation. Radiology 1996;200:665-71.

[20] European Commission, 1999 Quality Criteria for Computed Tomography, EUR $16262 \mathrm{EN}$.

[21] Lucaya J, Piqueras J, Sotil J, et al. Low-dose high-resolution CT of the chest in children and young adults: dose, cosoperation, artifact incidence, and image quality. AJR 2000;75:985-92. 